\title{
CONFORMAL SCALARS
}

\author{
BY JACK LEVINE
}

1. Introduction. In 1902, C. N. Haskins* determined the number of independent absolute scalar differential invariants of a given order of the general quadratic differential form. By the general theorem of Lie, $\dagger$ these invariants are given as the solutions of complete systems of partial differential equations. In Haskins' work, the coefficients of the quadratic form and their derivatives were used as the independent variables.

Later, T. Y. Thomas and A. D. Michal, $\ddagger$ starting with a relative quadratic differential form of weight $w \neq-2 / n$, showed how it could be reduced to an absolute quadratic form, and by using the coefficients of this form and the components of their tensor extensions as independent variables, obtained the differential equations for the scalar invariants and also their number for a given order. Their work was considerably simpler than Haskins', due to the choice of independent variables in the differential equations.

In this paper, the relative quadratic differential form of weight $w=-2 / n$ is considered, this being the exceptional case of the previous work. This form is associated with conformal geometry $\S$ and hence we need only consider $n \geqq 3$.

By a conformal scalar of order $r$ of a metric space will be meant a function\|

* Transaction of this Society, vol. 3 (1902), pp. 71-91. This paper will be called $\mathrm{H}$.

$\dagger$ Berichte der Gesellschaft der Wissenschaften zu Leipzig, vol. 43 (1891), pp. 316-393. TM.

$\ddagger$ Annals of Mathematics, vol. 28 (1927), pp. 631-687. We call this paper

$\S$ For a general discussion of conformal geometry, see T. Y. Thomas, Differential Invariants of Generalized Spaces, Cambridge University Press, 1934. This reference will be called $\mathrm{T}$.

$\|$ Throughout this paper all indices take the values $1, \cdots, n$ unless otherwise indicated. 


$$
{ }^{0} S\left(G_{i j} ; \frac{\partial G_{i j}}{\partial x^{k_{1}}} ; \cdots ; \frac{\partial^{r} G_{i j}}{\partial x^{k_{1}} \cdots \cdot \partial x^{k_{r}}}\right)
$$

which transforms by

${ }^{0} S\left(\bar{G}_{i j} ; \cdots ; \frac{\partial^{r} \bar{G}_{i j}}{\partial \bar{x}^{k_{1}} \cdots \partial \bar{x}^{k_{r}}}\right)={ }^{0} S\left(G_{i j} ; \cdots ; \frac{\partial^{r} G_{i j}}{\partial x^{k_{1}} \cdots \partial x^{k_{r}}}\right)$,

under an arbitrary coordinate transformation. The $G_{i j}$ are the components of the fundamental conformal relative tensor of weight $-2 / n$ and satisfy the condition $\left|G_{i j}\right|=1$.

2. The Differential Equations. To obtain the complete* system of differential equations satisfied by the conformal scalars of order $r$ we proceed as in $\mathrm{H}$ and find that the system is obtained by equating to zero the coefficients of the various derivatives of the $\xi^{i}$ in

where

$$
\sum_{p=0}^{r} X\left(\frac{\partial^{p} G_{i j}}{\partial x^{k_{1}} \cdots \partial x^{k_{p}}}\right) \frac{\partial^{0} S}{\partial\left(\frac{\partial^{p} G_{i j}}{\partial x^{k_{1}} \cdots \partial x^{k_{p}}}\right)}=0
$$

$$
\begin{aligned}
X\left(\frac{\partial^{p+1} G_{i j}}{\partial x^{a_{1}} \cdots \partial x^{a_{p}} \partial x^{a_{p+1}}}\right)= & \frac{\partial}{\partial x^{a_{p+1}}}\left[X\left(\frac{\partial^{p} G_{i j}}{\partial x^{a_{1}} \cdots \partial x^{a_{p}}}\right)\right] \\
& +\frac{\partial^{p+1} G_{i j}}{\partial x^{s} \partial x^{a_{1}} \cdots \partial x^{a_{p}}} \frac{\partial \xi^{s}}{\partial x^{a_{p+1}}} \\
X\left(G_{i j}\right)= & G_{i s} \frac{\partial \xi^{s}}{\partial x^{j}}+G_{s j} \frac{\partial \xi^{s}}{\partial x^{i}}-\frac{2}{n} G_{\imath j} \frac{\partial \xi^{s}}{\partial x^{s}} .
\end{aligned}
$$

The resulting differential equations will be called the equations of order $r$ and will be denoted by $E_{r n}, n$ being the dimension number of the space. From (1) it is seen that $E_{r+1, n}$ are obtained by adding terms to $E_{r n}$ and by adding new equations, which with Haskins we call the final equations of order $r+1$, and denote them by $F_{r+1, n}$.

As in $\mathrm{H}$, pp. 76-78, we prove the following theorem.

* That these equations are complete follows from Lie's theorem mentioned above. 
THEOREM 1. If $E_{r n}$ are independent, and if $F_{r+1, n}$ are independent, then $E_{r+1, n}$ are independent. If $F_{r+1, n}$ are dependent, then $F_{r n}$ are dependent.

By a slight modification of a proof in $\mathrm{H}$, pp. 81-82, we can prove the following theorem.

THEOREM 2. If $F_{2 n}$ are dependent, then $F_{2, n-1}$ are dependent.

The dependence of $F_{2 n}$ implies that

$$
G_{u s} \xi_{b c d}^{s}+G_{b \xi} \xi_{a c d}^{s}-\frac{2}{n} G_{a b} \xi_{s c d}^{s}=0,
$$

have solutions $\xi$ not all zero, the $\xi$ 's being symmetric in their lower indices. From (3) it is easily shown (by using $G_{i j}=\delta_{j}{ }^{i}$ ) that the following result is true.

TheOREM 3. $F_{23}$ are independent.

From Theorems 1, 2, and 3 we have the following theorem.

THEOREM 4. $F_{r n}$ are independent for $r \geqq 2, n \geqq 3$.

3. The Scalars for $n>3$. It is now necessary to find values of $n$ and $r$ for which $E_{r n}$ are independent. We shall use a combination of the methods of $\mathrm{H}$ and TM. We let $D_{r n}$ represent the difference in the number of independent variables and equations in $E_{r n}$. Then, if $E_{r n}$ are independent, $D_{r n}$ represents the number of independent conformal scalars of order $r$ for $n$ dimensions.

We first consider the case $r=2$. By evaluating the conformal scalar

$$
{ }^{0} S\left(G_{i j} ; \frac{\partial G_{i j}}{\partial x^{k}} ; \frac{\partial^{2} G_{i j}}{\partial x^{k} \partial x^{l}}\right)
$$

at the origin of conformal normal coordinates of order three, it is found that

$$
{ }^{0} S={ }^{0} T\left(G_{i j},{ }^{0} B_{i j k l}\right),
$$

where

$$
{ }^{0} B_{i j k l}=G_{i s}{ }^{0} B_{j k l}^{\boldsymbol{k}},
$$

and where ${ }^{0} B_{j k l}$ are the components of the Weyl conformal 
curvature tensor. ${ }^{*}$ The ${ }^{0} B_{i j k l}$ transform as the components of a relative tensor of weight $-2 / n$ and hence the differential equations which ${ }^{\circ} T$ satisfy are given by $\dagger$

$$
X_{s}^{t}(2, n)^{0} T \equiv\left(\begin{array}{c}
t \\
i j s
\end{array}\right) \frac{\partial^{0} T}{\partial G_{i j}}+\left(\begin{array}{r}
t \\
i j k l s
\end{array}\right) \frac{\partial^{0} T}{\partial^{0} B_{i j k l}}=0
$$

where

$$
\begin{aligned}
\left(\begin{array}{r}
t \\
i j s
\end{array}\right) & =G_{s j} \delta_{i}{ }^{t}+G_{i s} \delta_{j}^{t}-\frac{2}{n} G_{i j} \delta_{s}^{t} \\
\left(\begin{array}{r}
t \\
i j k l s
\end{array}\right)= & { }^{0} B_{s j k l} \delta_{i}^{t}+{ }^{0} B_{i s k l} \delta_{j}^{t}+{ }^{0} B_{i j s l} \delta_{k}^{t}+{ }^{0} B_{i j k s} \delta_{l}{ }^{t} \\
& \quad-\frac{2}{n}{ }^{0} B_{i j k l} \delta_{s}^{t} .
\end{aligned}
$$

In a manner similar to that used in TM, pp. 665-666 (proof of Theorem 11. II), we can prove the following theorem.

Theorem 5. If $X_{s}^{t}(2, n)=0$ are dependent, then $X_{s}^{t}(2, n-1)=0$ are dependent.

In this proof the components ${ }^{0} B_{i j k l}$ play the role of the second extension components $H_{\alpha \beta, \gamma \delta}$ of TM. For the ${ }^{0} B$ components a complete set of identities is given in [2.1] of C. In the proof of Theorem 5 we select among the dependent components

$$
G_{n 2}, \quad{ }^{0} B_{n 1 n 1}, \quad{ }^{0} B_{1 \alpha 1 \beta}, \quad(\alpha, \beta=1, \cdots, n-1) .
$$

We consider now

$$
X_{s}^{t}(2,4)=0 .
$$

In these equations there are 9 independent $G_{i j}$ and 10 independent ${ }^{0} B_{i j k l}$ (see C), and hence $X_{s}^{t}(2,4)=0$ consists of 16 equations in 19 independent variables. It is not difficult to show that these equations are independent, and so from this result and Theorem 5 we have the next theorem.

Theorem $6 . X_{s}^{t}(2, n)=0$ are independent for $n \geqq 4$.

* J. Levine, New identities in conformal geometry, Duke Mathematical Journal, vol. 1 (1935). We refer to this paper as C.

$\dagger$ See TM, p. 662, for a similar derivation. 
If we denote by $N(n, r)$ the number of functionally independent conformal scalars of order $r$ for $n$ dimensions, then from results of $\mathrm{C}$ and Theorem 6 we have

$$
\begin{aligned}
N(n, 2) & =\left(\frac{n(n+1)}{2}-1\right)+\frac{1}{12} n(n+1)(n+2)(n-3)-n^{2} \\
& =\frac{1}{12}\left(n^{4}-13 n^{2}-12\right), \quad(n \geqq 4) .
\end{aligned}
$$

It is found that $D_{2 n}$ is $N(n, 2)-n,(n \geqq 4)$, but since by Theorem $6, E_{2 n}$ must have $N(n, 2)$ solutions, this proves the following theorem.

TheOREM 7. There are $n$ dependent equations in $E_{2 n},(n \geqq 4)$.

Thus we must go to $E_{3 n}$ at least to find an independent set. The conformal scalar of order three is given by

$$
{ }^{0} S\left(G_{i j} ; \frac{\partial G_{i j}}{\partial x^{k}} ; \frac{\partial^{2} G_{i j}}{\partial x^{k} \partial x^{l}} ; \frac{\partial^{3} G_{i j}}{\partial x^{k} \partial x^{l} \partial x^{m}}\right) .
$$

By evaluating ${ }^{0} S$ at the origin of conformal normal coordinates of order four, we obtain*

$$
{ }^{0} S={ }^{0} T\left(G_{i j} ;{ }^{0} B_{i j k l} ;{ }^{0} B_{i j k l, m}\right),
$$

where ${ }^{0} B_{i j k l, m}$ represents components of the covariant derivative of $\dagger^{\circ} B_{i j k l}$. These components transform by

$$
\begin{aligned}
{ }^{0} \bar{B}_{a b c d, e}= & \left|\frac{\partial x}{\partial \bar{x}}\right|^{-2 / n}\left[{ }^{0} B_{i j k l, m} u_{a}^{i} u_{b}^{j} u_{c}^{k} u_{d}^{l} u_{e}^{m}\right. \\
& +\frac{1}{n}{ }^{0} B_{i j k l}\left(u_{a}^{0} u_{b}^{i} u_{c}^{l} u_{d^{k}} u_{e}^{j}-u_{b}^{0} u_{a}^{i} u_{c}^{l} u_{d^{k}} u_{e}^{j}\right. \\
& -u_{c}^{0} u_{a}^{i} u_{b}^{j} u_{d}^{k} u_{e}^{l}+u_{d}^{0} u_{a}^{i} u_{b}^{j} u_{c}^{k} u_{e}^{l} \\
& \left.\left.+2 u_{e}^{0} u_{a}^{i} u_{b}^{j} u_{c}^{k} u_{d}^{l}\right)\right], u_{j}^{i}=\frac{\partial x^{i}}{\partial \bar{x}^{j}}, \quad u_{j}{ }^{0}=\frac{\partial \log \left|\frac{\partial x}{\partial \bar{x}}\right|}{\partial \bar{x}^{j}} .
\end{aligned}
$$

We find that the differential equations satisfied by ${ }^{\circ} \mathrm{T}$ are given by the relations

* This follows from results of C.

† See T, p. 74. 


$$
\begin{aligned}
X_{s}^{t}(3, n)^{0} T \equiv & \left(\begin{array}{r}
t \\
i j s
\end{array}\right) \frac{\partial^{0} T}{\partial G_{i j}}+\left(\begin{array}{r}
t \\
i j k l s
\end{array}\right) \frac{\partial^{0} T}{\partial^{0} B_{i j k l}} \\
& +\left(\begin{array}{r}
t \\
i j k l m s
\end{array}\right) \frac{\partial^{0} T}{\partial^{0} B_{i j k l, m}}=0
\end{aligned}
$$

(6) $X^{t}(3, n)^{0} T \equiv\left[\begin{array}{r}t \\ i j k l m\end{array}\right] \frac{\partial^{0} T}{\partial^{0} B_{i j k l, m}}=0$, where

$$
\begin{gathered}
\left(\begin{array}{r}
t \\
i j k l m s
\end{array}\right)={ }^{0} B_{s j k l, m} \delta_{i}{ }^{t}+\cdots+{ }^{0} B_{i j k l, s} \delta_{m}{ }^{t}-\frac{2}{n}{ }^{0} B_{i j k l, m} \delta_{s}{ }^{t} \\
{\left[\begin{array}{r}
t \\
i j k l m
\end{array}\right]={ }^{0} B_{m j k l} \delta_{i}{ }^{t}+{ }^{0} B_{i m k l} \delta_{j}{ }^{t}+{ }^{0} B_{i j m l} \delta_{k}{ }^{t}} \\
+{ }^{0} B_{i j k m} \delta_{l}{ }^{t}+2{ }^{0} B_{i j k l} \delta_{m}{ }^{t} .
\end{gathered}
$$

From (4) and Theorem 6, and the form of (5) and (6), we see that (5) and (6) are independent if equations (6) are. By use of the complete set of identities for $G_{i j},{ }^{0} B_{i j k l},{ }^{0} B_{i j k l, m}$, we can show that (6) are independent. Hence we have the following theorem.

Theorem 8. The equations $X_{s}^{t}(3, n)=0, X^{t}(3, n)=0$ for the determination of the conformal scalars of order three for $n \geqq 4$ are independent.

The number of independent components $G_{i j},{ }^{0} B_{i j k l},{ }^{0} B_{i j k l, m}$ is given by

$$
\begin{aligned}
A=[K(n, 2)-1] & +\frac{1}{12} n(n+1)(n+2)(n-3) \\
& +\lfloor K(n, 2) K(n, 3)-n K(n, 4)-K(n, 3)],
\end{aligned}
$$

where

$$
K(n, a)=\frac{n(n+1) \cdots(n+a-1)}{a !}, \quad K(n, 0)=1 .
$$

Hence

$$
\begin{aligned}
N(n, 3)=A-\left(n^{2}+n\right)=\frac{1}{24}[ & (n+2)\left(n^{4}+2 n^{3}-9 n^{2}+2 n\right. \\
& \left.-12)-24 n^{2}-24 n\right], \quad(n \geqq 4) .
\end{aligned}
$$


It is found that $D_{3 n}$ is also $N(n, 3)$, thus proving the following theorem.

THEOREM 9. $E_{3 n}$ are independent for $n \geqq 4$.

We can now state the main theorem which follows from Theorems 1 and 4 .

THEOREM 10. The equations $E_{r n}$ for the determination of the conformal scalars for $r \geqq 3$ and $n \geqq 4$ are independent.

We find that

$$
D_{r n}=N(n, r)=[K(n, 2)-1] \sum_{a=0}^{r} K(n, a)-n \sum_{a=1}^{r+1} K(n, a)
$$

for $r \geqq 3$ and $n \geqq 4$. By means of the relations

$$
K(n, a)-K(n, a-1)=K(n-1, a), \sum_{a=0}^{r} K(n, a)=K(n+1, r),
$$

we obtain

$$
N(n, r)=n+\left(\frac{n^{2}+n-2}{2}-\frac{n(n+r+1)}{r+1}\right) \frac{(n+r) !}{n ! r !},
$$

for $r \geqq 3, n \geqq 4$.

4. The Scalars for $n=3$. For this case ${ }^{0} B_{i j k l}=0$. The set (5) and (6) consist now of 9 equations with 10 independent variables. These equations, $X_{s}^{t}(3,3)=0$, are independent, showing that $N(3,3)=1$. There are 102 equations and 100 independent variables in $E_{33}$ and as these equations must have one solution as has just been stated, it follows that $E_{33}$ contains 3 dependent equations. Thus we must try $r=4$ at least to determine an $r$ for which $E_{r 3}$ are independent.

If we denote by ${ }^{0} G_{i j}(y)$ the component $G_{i j}$ in conformal normal coordinates of order $p$, we can define quantities

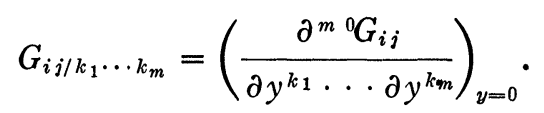

For $p=5$, we can determine a complete set of identities for $G_{a b / c d e f}$. Such a set is given by 
$G_{a b / c d e f}=G_{b a / c d e f}=G_{a b / i j k l}, \quad(i j k l=$ any permutation of $c d e f) ;$ $G^{a b} G_{a b / c d e f}=0 ; \quad G_{i a / b c d e}+G_{i b / c d e a}+G_{i c / d e a b}+G_{i d / e a b c}+G_{i e / a b c d}=0$.

It is possible* to express the quantities $G_{i j / k l m p}$ in terms of the

$$
G_{a b} ;{ }^{0} B_{a b c d, e} ;{ }^{0} B_{a b c d, e, f} ;{ }^{0} B_{a b c d, \infty} ;{ }^{0} B_{a b \infty}^{0} \text {. }
$$

Hence by transforming the conformal scalar ${ }^{0} S$ of order 4 to conformal normal coordinates of order 5 , and evaluating at the origin, we find

$$
{ }^{0} S={ }^{0} U\left(G_{i j} ;{ }^{0} B_{i j k l, m} ;{ }^{0} B_{i j k l, m, p} ;{ }^{0} B_{i j k l, \infty} ;{ }^{0} B_{i j \infty}^{0}\right) .
$$

From the laws of transformation of the above components we find the differential equations satisfied by ${ }^{0} U$ to be

$$
\begin{aligned}
X_{s}{ }^{t}(4,3)^{0} U \equiv & \left(\begin{array}{c}
t \\
i j s
\end{array}\right) \frac{\partial^{0} U}{\partial G_{i j}}+\left(\begin{array}{r}
t \\
i j k l m s
\end{array}\right) \frac{\partial^{0} U}{\partial^{0} B_{i j k l, m}} \\
& +A_{i j k l m p s}^{t} \frac{\partial^{0} U}{\partial^{0} B_{i j k l, m, p}}+C_{i j k l s}^{t} \frac{\partial^{0} U}{\partial^{0} B_{j k l, \infty}} \\
& +D_{i j s}^{t} \frac{\partial^{0} U}{\partial^{0} B^{0} j \infty}=0,
\end{aligned}
$$

$$
\begin{aligned}
X^{t}(4,3)^{0} U \equiv & G^{m p} G^{k t}{ }^{0} B_{m k a b, p} \frac{\partial^{0} U}{\partial^{0} B_{a b \infty}} \\
& +E_{a b c d}^{t} \frac{\partial^{0} U}{\partial^{0} B_{a b c d, \infty}}+F_{a b c l e f}^{t} \frac{\partial^{0} U}{\partial^{0} B_{a b c d, e, f}}=0 .
\end{aligned}
$$

The quantities $A, C, D, E, F$, of (7) and (8) are certain functions of the variables present, and their exact form is not needed. The equations (7) are independent since $X_{s}^{t}(3,3)=0$ are. By means of the relations ${ }^{0} B_{k a b}^{0}=-G^{m p}{ }^{0} B_{m k a b, p},(n=3)$, (proved in C) we can show (8) are independent, and hence (7) and (8) are also.

THEOREM 11. The equations $X_{s}^{t}(4,3)=0, X^{t}(4,3)=0$ for the determination of the conformal scalars of order 4 for $n=3$ are independent.

* For the definitions of the new components see T, pp. 75, 81 . 
The number of independent components $G_{i j / k l m p}$ is $K(3,2) K(3,4)-3 K(3,5)-K(3,4)=12$, which is also the number of independent components ${ }^{0} B_{i j k l, m, p} ;{ }^{0} B_{i j k l, \infty} ;{ }^{0} B_{i j \infty}$. Thus equations (7) and (8) have 10 solutions. Now $D_{43}$ is also 10, (175-165). Hence we have the following theorem.

THEOREM 12. $E_{43}$ are independent.

Finally, from Theorems 1 and 4, we have the following theorem.

THEOREM 13. $E_{r 3}$ are independent for $r \geqq 4$.

We find

$$
N(3, r)=3+\frac{1}{6}(2 r-7)(r+2)(r+3), \quad(r \geqq 4) .
$$

5. Scalars of Orders Zero and One. For the conformal scalars of order zero we have the equations

$$
\text { (9) } \begin{aligned}
Y_{s}{ }^{t}(0, n)^{0} T \equiv\left(G_{s j} \delta_{i}{ }^{t}+G_{i s} \delta_{j}{ }^{t}-\frac{2}{n} G_{i j} \delta_{s}{ }^{t}\right) & \frac{\partial^{0} T}{\partial G_{i j}}=0, \\
& (i \leqq j, i \cdot j \neq 1),
\end{aligned}
$$

where the dependent components are to be $G_{11} ; G_{i j},(i>j)$. By dividing up the equations (9) into sets represented by

$$
X_{t-1}^{t}=0, X_{t+1}^{t}=0, \cdots, X_{n}^{t}=0, \quad(t=1,2, \cdots, n),
$$

we can show by a process of induction that $\partial^{0} T / \partial G_{i j}=0$, $(i \leqq j, i \cdot j \neq 1)$. The first step is to show that $X_{s}^{t}(0, n)=0 \mathrm{im}$ ply $\partial^{0} T / \partial G_{1 j}=0,(j=2, \cdots, n)$. Then by assuming $\partial^{0} T / \partial G_{i t}=0$, $(i=1, \cdots, t-1)$, we show $\partial^{0} T / \partial G_{t j}=0,(j=t, \cdots, n)$.

If the conformal scalar ${ }^{0} S\left(G_{i j} ; \partial G_{i j} / \partial x^{k}\right)$ of order one be evaluated at the origin of a system of conformal normal coordinates of order two, it is found that ${ }^{0} S={ }^{0} S\left(G_{i j} ; 0\right)$. Combining these results we have the following theorems.

THEOREM 14. There are no conformal scalars of order zero or of order one.

THEOREM 15. There are no conformal scalars of order two for $n=3$. 
For if we consider (4) for $n=3$, we see that these equations reduce to (9).

We give here a summary of the results obtained concerning the number of functionally independent conformal scalars of a given order.

$$
\begin{array}{lr}
N(n, r)=n+\left(\frac{n^{2}+n-2}{2}-\frac{n(n+r+1)}{r+1}\right) \frac{(n+r) !}{n ! r !}, \\
(n \geqq 4, r \geqq 3), \\
N(n, 2)=\frac{1}{12}\left(n^{4}-13 n^{2}-12\right), & (n \geqq 4), \\
N(3, r)=3+\frac{1}{6}(2 r-7)(r+2)(r+3), & (r \geqq 4), \\
N(3,3)=1, & (n \geqq 3) . \\
N(n, 0)=N(n, 1)=N(3,2)=0, &
\end{array}
$$

We may construct a conformal scalar as follows. We put

$$
T=G^{a b} G^{c d}{ }^{0} B_{i a c}^{h} B_{h b d}^{i} ; \quad U=G^{a b} G^{d e} G^{i j 0} B_{m j c b}{ }^{0} B_{i d a}^{m} .
$$

Then the ratio $T / U$ is the scalar. The quantities $T$ and $U$ are relative conformal scalars.

It is to be noted that conformal normal coordinates of order $r$ are analogous to geodesic coordinates of order $r$ in affine geometry.*

North Carolina State College

* A. D. Michal, Geodesic coordinates of order $r$, this Bulletin, vol. 36 (1930), pp. 541-546. 